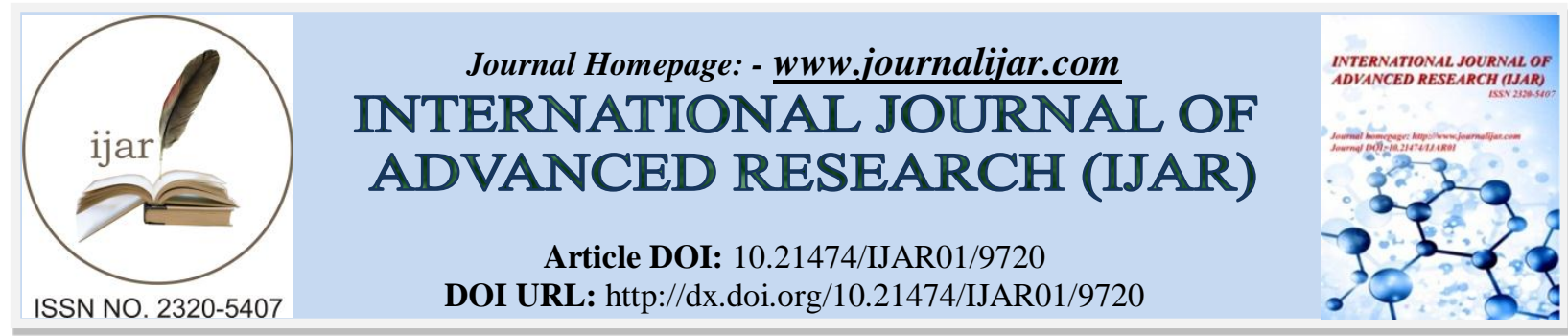

RESEARCH ARTICLE

\title{
REGLES TRADITIONNELLES DE PROTECTION DES RESSOURCES EN EAU DANS LE BASSIN VERSANT DE LA MEKROU (SOUS BASSIN BÉNINOIS DU FLEUVE NIGER, AFRIQUE DE L'OUEST).
}

\author{
Ali moumouni bawa n'gobi ${ }^{1}$, michel boko ${ }^{2}$, E.W. Vissin ${ }^{2}$ and C. S. Hedible ${ }^{2}$. \\ 1. Département de Géographie et Aménagement du Territoire FASHS/UAC, Bénin. \\ 2. Laboratoire d'étude des climats, des Ressources en Eau et de la Dynamique des Ecosystèmes (LECREDE), \\ Cotonou, Bénin.
}

\section{Manuscript Info}

Manuscript History

Received: 09 July 2019

Final Accepted: 12 August 2019

Published: September 2019

Key words:-

Mekrou, watershed, water, endogenous

knowledge, integrated management.

\section{Abstract}

The agricultural, hunting and fishing potentials of the Mékrou watershed have favored the establishment of different socio-cultural groups that perceive water as a god's gift. And as such, it is a common good of communities. To better understand this question, the perception of the populations of the origin of the water and of the hydro-climatic variation in the basin was studied.

The methodological approach used includes data collection, processing and analysis. Socio-economic surveys were conducted in selected villages on the basis of well-defined criteria. In total, 271 people were surveyed during the fieldwork that was conducted in 34 localities of the Mékrou watershed.

The results of this research show that, for all the populations of the Mekrou basin, water has a cultural and cultic dimension. It would house deities. The water is sacred and its protection is through the use of these deities through invocations, the practice of rituals, cults and the introduction of prohibitions etc according to the people surveyed. In general, the perceptions of pre-colonial societies in Benin of the management and protection of water resources are mentioned in the "customary of Dahomey". Nowadays, because of the increase in the population, the frantic race for profit, the proliferation of beliefs, the disappearance of points, plans and rivers due to climate change and to anthropogenic actions, the endogenous practices of protection of water resources are becoming increasingly stale. Also, through their behavioral reflexes, the populations show that they skilfully ignore everything of the modern legal and institutional mechanism set up for the enjoyment and rational management of these water resources. The modern rules of water resources management are not, in the current state of affairs, (the illiteracy of the populations fundamentally anchored in their socio-cultural realities) will not be able to assure their real protections. So, a good protection of these resources goes through the combination of these endogenous protection strategies and the legal instruments both national and international applied with rigor.

Copy Right, IJAR, 2019,. All rights reserved. 


\section{Introduction:-}

Dans la cosmogonie traditionnelle locale des populations du bassin, l'eau occupe une place de choix après la terre et l'air. Vient ensuite le feu. L'eau est un don de dieu et par conséquent un bien commun. Son accès est libre et gratuit. Dans le Coutumier du Dahomey, la question de l'eau est réglée aux points 223 et 224. Il s'agit principalement de la propriété collective et du caractère sacré et inaliénable de l'eau.

Le point 223 précise : «la propriété collective du village comprend des terrains indivis, sensés appartenir personnellement au Roi, entretenus par le village et sur lesquels tous ont droit de culture, pacage, affouage, de chasse et de pêche. Enfin des sources, des puits. Ces biens sont inaliénables, mais peuvent cependant être loués ou prêtés » (MOUMOUNI A. B. N., 2007, p 11). Le point 224 du Coutumier parle, lui du « domaine public religieux» de l'eau. «il existe enfin une espèce de domaine public religieux : bois, rivières, lieux saints, terrains servant à l'inhumation (en cas de mort par maladie contagieuse) inaliénables et sacrés » (MOUMOUNI A. B. N., 2007, p 11). Ce caractère sacré et religieux de l'eau est réaffirmé par DEBOUROU D., (2012, p 319) dans la société baatonnu du Nord-Bénin son passé, son dynamisme, ses conflits et ses innovations. «Le panthéon baatonnu se compose de nombreux dieux symbolisés par un bloc de pierre, un marigot, une rivière, une source, un arbre, les ancêtres morts, un reptile ou un mammifère... » (DEBOUROU, D., 2012, p 319)

Malgré le caractère libre et gratuit de l'accès à l'eau, des dispositions sont prises pour lui éviter des désagréments. C'est ce qui explique l'instauration des totems, des interdits liés à l'eau. Ces dispositions endogènes non écrites sont prises non seulement pour protéger les ressources déjà disponibles mais aussi pour faire en sorte que les pluies tombent lorsque l'eau viendrait à manquer. La présente recherche a pour objectif de mettre en exergue, chez les différents groupes socio-culturels établis dans le bassin, la contribution de leurs savoirs endogènes à la protection des ressources en eau de ce dernier.

\section{Materiel Et Methodes:-}

\section{Zone d'étude}

La mékrou, l'objet de cette recherche draine un bassin versant de forme très allongée d'environ $10.690 \mathrm{~km}^{2}$ dont les $2000 \mathrm{~km}^{2}$ sont au Burkina-Faso et en république du Niger (Thiebaux, 1985 ; A., 2009). Au Bénin, il traverse cinq communes à savoir : Kouandé, Kérou, Péhunco, Banikoara et Karimama. Il prend sa source à 3 km au Nord-est du village de Yakabissi dans l'arrondissement de Birni, commune de Kouandé. Sa confluence avec le fleuve Niger se situe à Mékrou-Tounga soit à $3 \mathrm{~km}$ du village de Pétchinga dans l'arrondissement de Monsey (Commune de Karimama).

Le bassin Culmine environ 610 m d'altitude et se situe entre $10^{\circ} 02^{\prime}$ et $12^{\circ} 17^{\prime}$ de latitude Nord et entre $1^{\circ} 34^{\prime}$ et $2^{\circ}$ 51 ' de longitude Est. La figure 1 présente la situation géographique du bassin versant de la Mékrou.

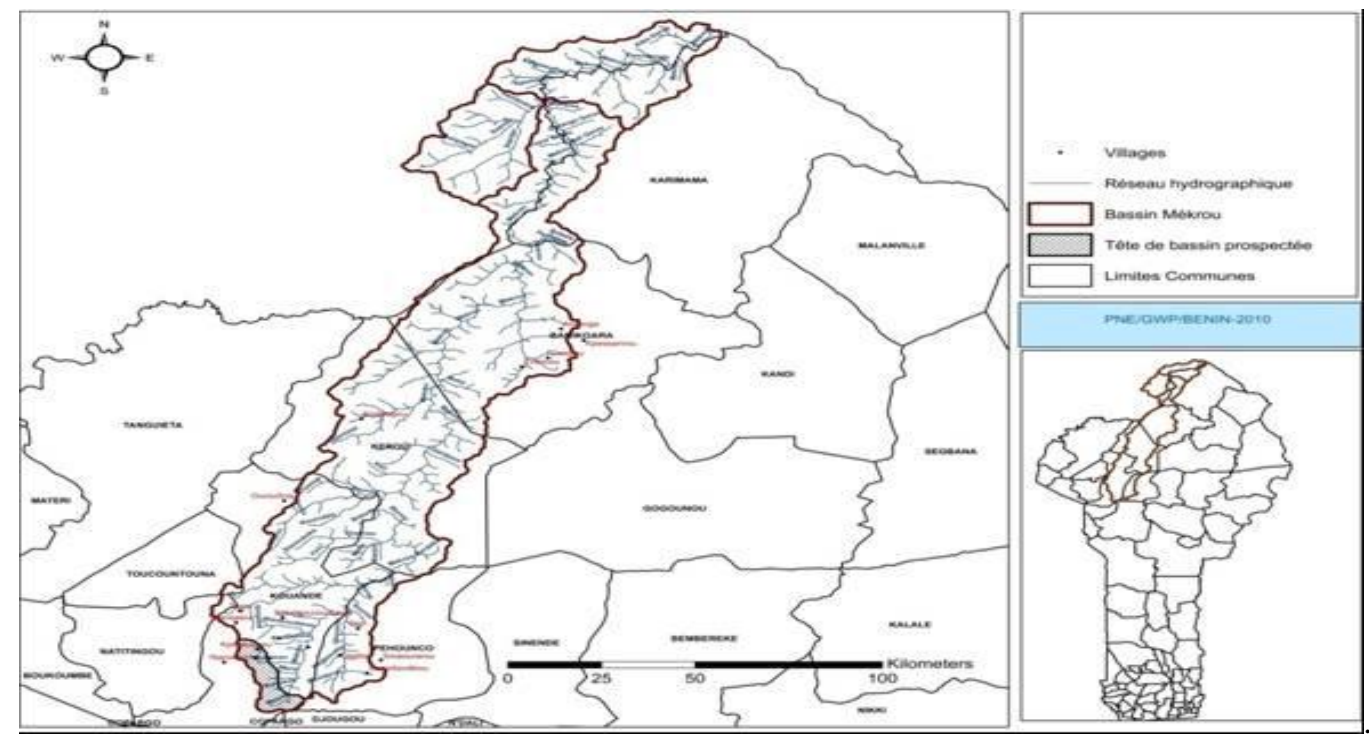

Figure 1:-Situation géographique du bassin versant de la Mékrou 


\section{Méthode:-}

La méthodologie utilisée pour la présente recherche a consisté à la collecte des données, leur traitement et leur analyse.

\section{Collecte des données et informations}

La recherche sur les savoirs endogènes de protection des ressources en eau du bassin de la Mékrou revêt une nature qualitative. En effet, elle va produire et analyser des données relatives aux discours, aux pratiques, aux perceptions, des différents groupes socio-culturels établis dans le bassin, des ressources en eau, des stratégies de protection de ces ressources et de la variabilité climatique. Pour y parvenir, il a été nécessaire de disposer de données en lien avec la disponibilité des eaux atmosphériques, des eaux superficielles ainsi que celles des eaux souterraines. Les données climatologiques et de population ont été également recueillies comme l'indiquent plus haut les tableaux dans la partie présentation du cadre géographique du secteur d'étude. A la recherche documentaire se sont ajoutées des enquêtes de terrain sur la base d'un Questionnaire d'enquête sociologique préétabli. Ces enquêtes ont permis une meilleure appréciation de la perception des populations du bassin des ressources en eau, de leur protection et de la variabilité climatique.

Au total, 271 personnes ont été enquêtées lors des travaux de terrain qui ont été conduits dans 34 localités du bassin versant de la Mékrou. Les localités du bassin situées à proximité du cours d'eau principal ou de l'un de ses importants affluents, celles abritant des ouvrages de retenue d'eau comme les barrages ou des sources d'eau naturelles ont été priorisées et ce sur la base d'un choix raisonné.

\section{Traitement et analyses des données}

Les données qualitatives issues des discussions de groupe ont servi de base pour l'analyse des principaux résultats obtenus. Les données issues de l'enquête ont été codifiées puis saisies dans une matrice de gestion de base de données à l'aide du tableur Excel version 2013.

\section{Resultats:-}

Perceptions des populations de l'origine de l'eau et de la variation hydro climatique dans le bassin Origine des ressources en eau

Les différentes ressources en eau du bassin sont : les eaux pluviales, les eaux superficielles et les eaux souterraines.

\section{Les eaux pluviales :}

Elles permettent de programmer les activités agricoles. Leur disponibilité marque le début d'une nouvelle saison appelée saison pluvieuse qui dure entre cinq à six mois (de mai à octobre). Cette saison alterne avec une autre qui marque l'arrêt de la pluie et dure entre six à sept mois (mi-octobre à avril) qui est appelée saison sèche.

\section{Les eaux superficielles :}

Elles sont constituées par les cours d'eau, les marres, des barrages etc. Elles servent à plusieurs usages.

Les eaux souterraines :

Ce sont celles qui sont contenues dans les nappes aquifers L'origine des ressources en eau, quelles qu'elles soient, est souvent attribuée aux divinités

\section{Les variations hydro climatiques dans le bassin}

La dégradation de l'environnement en général (sols, eau, air) est assez remarquable dans le bassin. Il fait de plus en plus chaud en période de chaleur et de plus en plus froid en période d'harmattan. Les niveaux d'eau diminuent d'année en année. Les pluies sont tardives et s'arrêtent de façon précoce ou tardive.

Selon les personnes rencontrées, deux raisons fondamentales sont à l'origine de cette situation. Il s'agit de la contrariété des saisons et surtout des actions anthropiques telle que l'extension des cultures jusqu'au niveau des berges, l'utilisation intense des intrants chimiques dans l'agriculture et l'usage de ces produits dans la pêche etc. Mais il faut noter que le fléau le plus ahurissant reste le déboisement sans précédent des forêts aussi bien par les agriculteurs que par les exploitants forestiers. A ce niveau, les différents acteurs (autorités locales, agents des eaux et forêts, populations à la base) se jettent le tort les uns sur les autres. La planche 3 présente l'exploitation forestière aux abords et dans le lit de la Mékrou (Yakabissi) et dans le lit de Kourou, un de ses importants affluents à 
Nassoukou. La planche 1 illustre l'exploitation forestière dans le lit de la Mékrou à Yakabissi (photo 1) et de Kourou à Nassoukou (photo 2).

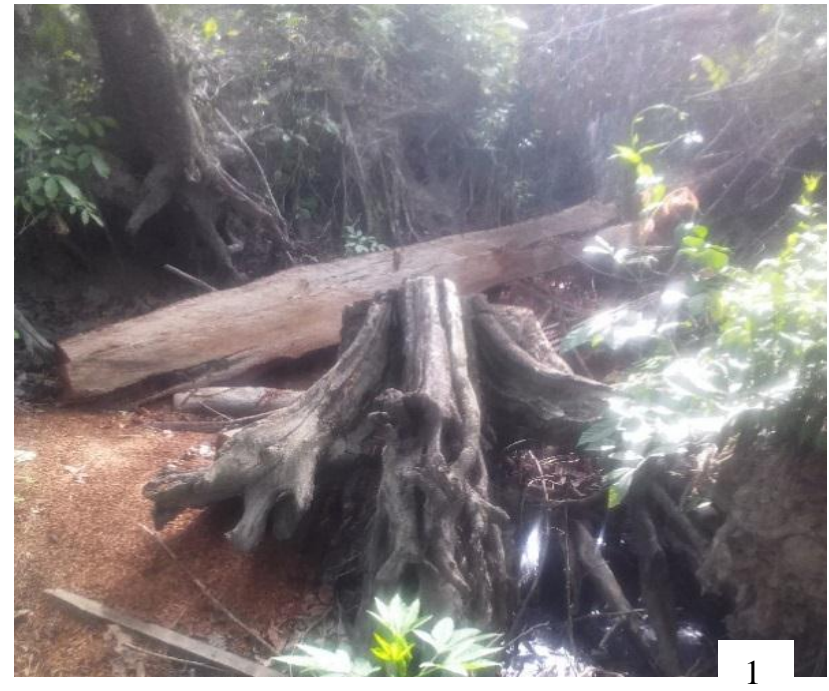

Photo 1 :-Exploitation forestière dans le lit de la Mékrou à Yakabissi

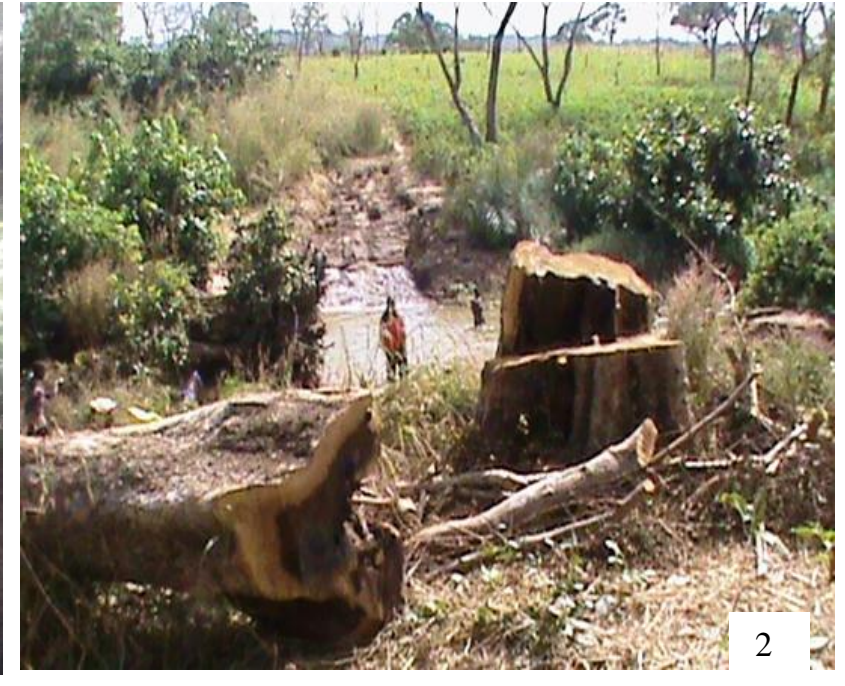

Photo 2:-Exploitation forestière dans le lit de Kourou (affluent de la Mékrou) à Nassoukou

\section{Planche 1 :}

Exploitation forestière dans le lit de la Mékrou à Yakabissi et de Kourou à Nassoukou.

\section{Prise de vue :}

Moumouni, Mai 2018

Ceci expliquerait donc le comblement ou l'ensablement accru des cours d'eau, voire la disparition totale de certains plans ou points d'eau. Le tableau I présente une synthèse sur la perception des populations sur la variation hydroclimatique dans le bassin versant de la Mékrou.

Tableau I :-Synthèse sur la perception des populations de la variation hydroclimatique dans le bassin versant de la Mékrou

\begin{tabular}{|c|c|}
\hline Observations & Manifestations/conséquences \\
\hline Le climat a changé & $\begin{array}{l}\text { Démarrage tardif et/ou la mauvaise répartition des pluies } \\
\text { Forte turbidité de la fréquence et l'intensité des pluies } \\
\text { D'après } 87 \% \text { des populations on assiste à la concentration de la pluie sur courte } \\
\text { période et une rupture précoce ou tardive. }\end{array}$ \\
\hline Poches de sécheresse & $\begin{array}{l}\text { Tarissement rapide des cours et plan d'eau } \\
\text { Ensablement et disparition des plans et points d'eau ou ressources stagnantes } \\
\text { Disparition de forêts galeries et des animaux sauvages }\end{array}$ \\
\hline $\begin{array}{l}\text { Raccourcissement de la durée } \\
\text { de saison pluvieuse }\end{array}$ & $\begin{array}{l}\text { L'arrivée tardive des pluies qui connaissent une fin précoce. Cela occasionne un } \\
\text { raccourcissement de la saison pluvieuse : «Avant, dans les années } 1970 \text { voire1980, } \\
\text { on n'avait } 5 \text { à } 6 \text { mois de pluies mais maintenant, on n'a que } 3 \text { à } 4 \text { mois ». } \\
\text { Selon } 67 \% \text { des enquêtés, les cultures locales qui ont généralement un cycle long ne } \\
\text { tiennent plus. Ceci conduit à l'envahissement des zones humides telles que les bas- } \\
\text { fonds et les berges. }\end{array}$ \\
\hline $\begin{array}{l}\text { Accroissement de la } \\
\text { transhumance }\end{array}$ & $\begin{array}{l}\text { La transhumance contribue à la dégradation des terres, à l'augmentation des } \\
\text { inondations, au surpâturage et aux conflits entre migrants autochtones. }\end{array}$ \\
\hline $\begin{array}{l}\text { Désacralisation des points, } \\
\text { plans et cours d'eau et forêts }\end{array}$ & $\begin{array}{l}\text { Cela constitue l'une des causes de l'empoisonnement des cours d'eau et la } \\
\text { dégradation des écosystèmes et de la biodiversité } \\
\text { Envahissement des points d'eau disponibles, conflits divers autour de l'eau. } \\
\text { La déforestation, la destruction des forets galerie l'utilisation abusive des engrais } \\
\text { minéraux, d'herbicide et de pesticide surtout dans la zone cotonnière constituée des }\end{array}$ \\
\hline
\end{tabular}


communes telles que Kouande, Péhunco, Kérou et Banikoara.

Source : enquête de terrain, mai 2018

L'analyse du tableau atteste que les populations riveraines du bassin versant de la mékrou perçoivent la variation des paramètres climatiques d'une part et leurs effets sur la disponibilité de ses ressources en eau d'autre part.

Parlant des changements climatiques et de la disparition des points, plans et cours d'eau au cours d'un entretien, Dame Bana Kyo BRISSO, une vieille octogénaire de Kèdékou dans la Commune de Kouandé nous a confié ceci :

L'homme possède sa case où il prend repos après une journée de dur labeur. Cette case met celui-ci à l'abri des intempéries. Lorsque celle-ci viendrait à être détruite, combien de temps ce dernier pourrait-il résister au soleil, à la pluie, à la chaleur et au froid ? .

De la même façon, si malgré sa générosité, les hommes sortent la Mékrou de son lit, la débarrassent de ses vêtements que sont les arbres et les buissons et lui déversent dessus des ordures excrémentielles, des produits mortels des blancs (engrais chimiques, pesticides, herbicides tel que le glyphosate), combien de temps peut-t-elle résister aux intempéries ? Et à quoi pourrait-elle être utile ? Peut-on vouer une institution sacrée aux gémonies ? Autant le bariba ou baatonnu préfêre la mort à la honte, autant la Mékrou ne pourrait survivre au déshonneur, à l'infamie.

Perception des populations de la protection des ressources en eau du basin Chez les différents groupes socioculturels du bassin de la Mékrou, l'eau est un don de dieu. Cette eau abriterait des divinités.

Alors sa gestion est confiée, dans les Communes de Kouandé, Péhunco et Kérou par le roi, au Ministre chargé des cultes qui est en même chef de terre. Ce Ministre est issu du groupe social le plus autochtone. Dans la Commune de Banikoara, le Chef lui-même préside les cultes. Le Ministre chargé des cultes et prêtre des eaux peut déléguer ses pouvoirs à des personnes relevant de son groupe social. Les cultes se font conformément aux prescriptions divinatoires du dieu donateur de l'eau.

Lors des enquêtes de terrain à Bonni (Commune de Kérou), la question a été posée de savoir pourquoi le fétiche Kpétara (pierres tressées) a été érigé au bord de la rivière Mékrou. A cette question, les populations ont répondu en ces termes:

1. «Ce sont les dieux qui ont choisi de s'installer à cet endroit précis. Les nuits, des chants et sons de tam tam nous parvenaient de cet endroit. Des recherches ont révélé l'existence des esprits, des divinités à cet endroit. C'est ainsi que Kpétara a été érigé là ».

2. La propriété commune des ressources en eau constituant la base des rapports intercommunautaires, il eut fallu édicter des règles de conduite pour une gestion efficace et efficiente desdites ressources.

3. Ainsi dans la plupart des localités parcourues les mesures suivantes sont prises :

4. Interdiction formelle de faire la vaisselle (laver les ustensiles de cuisine) aux abords des points, plans et cours d'eau.

5. Les femmes y sont interdites d'accès lorsqu'elles sont en menstrues.

6. Interdiction leur est également faite de laver aux abords des plans et points d'eau les graines de néré destinées à préparer de la moutarde. En effet, les eaux usées et résidus résultant du lavement de ces graines dégagent, dans leur décomposition, une odeur pestilentielle.

7. A Bonni, les dieux qu'incarne le fétiche «Kpétara» interdisent formellement de couper du bois de chauffe dans la forêt galerie bordant le cours d'eau. Toute femme qui s'obstine à le faire est frappée de stérilité ou si elle avait eu déjà des enfants ces derniers mourraient l'un après l'autre.

8. A un usage déterminé de l'eau correspond un endroit précis, identifié et séparé des autres endroits destinés à d'autres usages.( lieu de culte séparé du lieu de pêche et de ceux destinés au bain et à la vaisselle).

Le prêtre des eaux veille à l'application de ces règles de conduite élaborées pour une bonne gestion des ressources en eau. Les contrevenants s'exposent à des sanctions très sévères.

Dans les communes du bassin (Kouandé, Kérou, Péhunco et Banikoara) majoritairement peuplées de Baribas ou Baatombu, des cultes ont lieu aux abords de la rivière Mékrou dans les villages du bassin que traverse le cours d'eau. Ces cultes revêtent diverses formes (libations, offrandes, récitations de prières adressées à la divinité).

«Le panthéon baatonnu se compose de nombreux dieux symbolisés par un bloc de pierre, un marigot, une rivière, une source, un arbre, les ancêtres morts, un reptile ou un mammifère. C'est à eux qu'on voue des cultes, qu'on 
dédie des sacrifices qui revêtent plusieurs formes : des libations aux offrandes en passant par de simples récitations de prières adressées au pied de la divinité ». (Djibril DEBOUROU, 2012 Page 319) dans la société baatonnu du Nord-Bénin son passé, son dynamisme, ses conflits et ses innovations.

La planche 1 présente "Sarkaré » une source au pied de la montagne. Cette source est sacrée. Elle abrite une divinité représentée par un boa auquel le Ministre du culte, le Kouandé Sounon fait des libations et offre œufs, poulets, bœuf. Les interdits liés à l'usage de l'eau de cette source ont été évoqués plus haut. Ces interdits sont relatifs aux ustensiles de cuisine et aux graines de néré qui ne doivent pas être portés à la source pour y être lavés. La planche 2 montre Sarkaré, source naturelle d'eau aménagée à Kouandé centre.
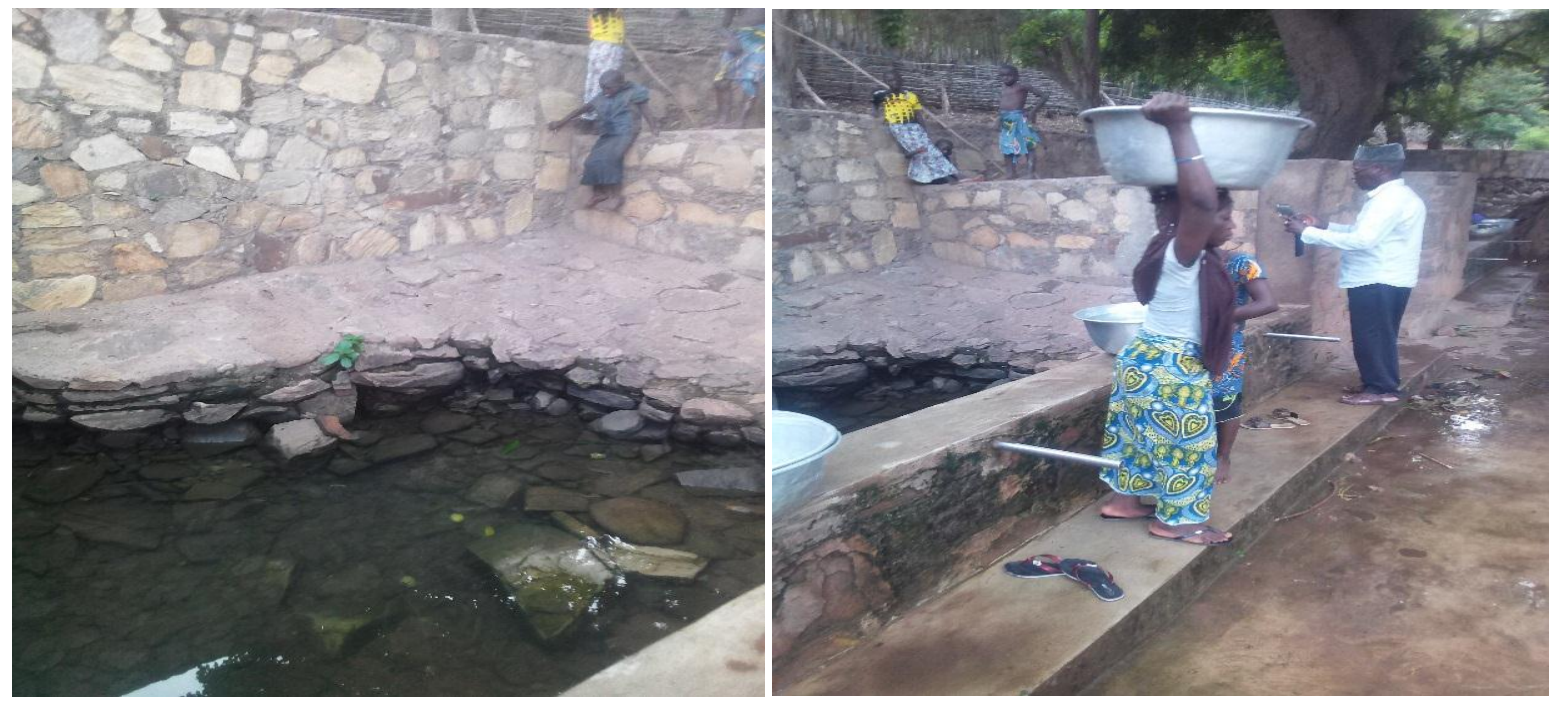

Planche 2:-Sarkaré, source naturelle d'eau aménagée à Kouandé centre.

\section{Prise de Vue : Moumouni, Mai 2018}

Sise au pied d'une montagne, cette source a, depuis la nuit des temps, alimenté les populations de Kouandé en eau de consommation. Son eau qui suinte du dessous des rochers est très appréciée et recherchée.

La planche 2 présente la chute de Koudou en plein cœur du parc W à la hauteur de Sampèto dans la commune de Banikoara. La première photo de la planche présente le site où les villageois, sous la direction de leur Chef, le Nansounon de Banikoara, pratiquent leurs rites sacrificiels. Même en saison sèche, l'endroit reste vert et l'eau descend tumultueusement le /long des sept rapides qui forment les chutes de Koudou (planche 3). En se promenant dans cet endroit magnifique, on comprend aisément l'attachement que les populations riveraines lui vouent.

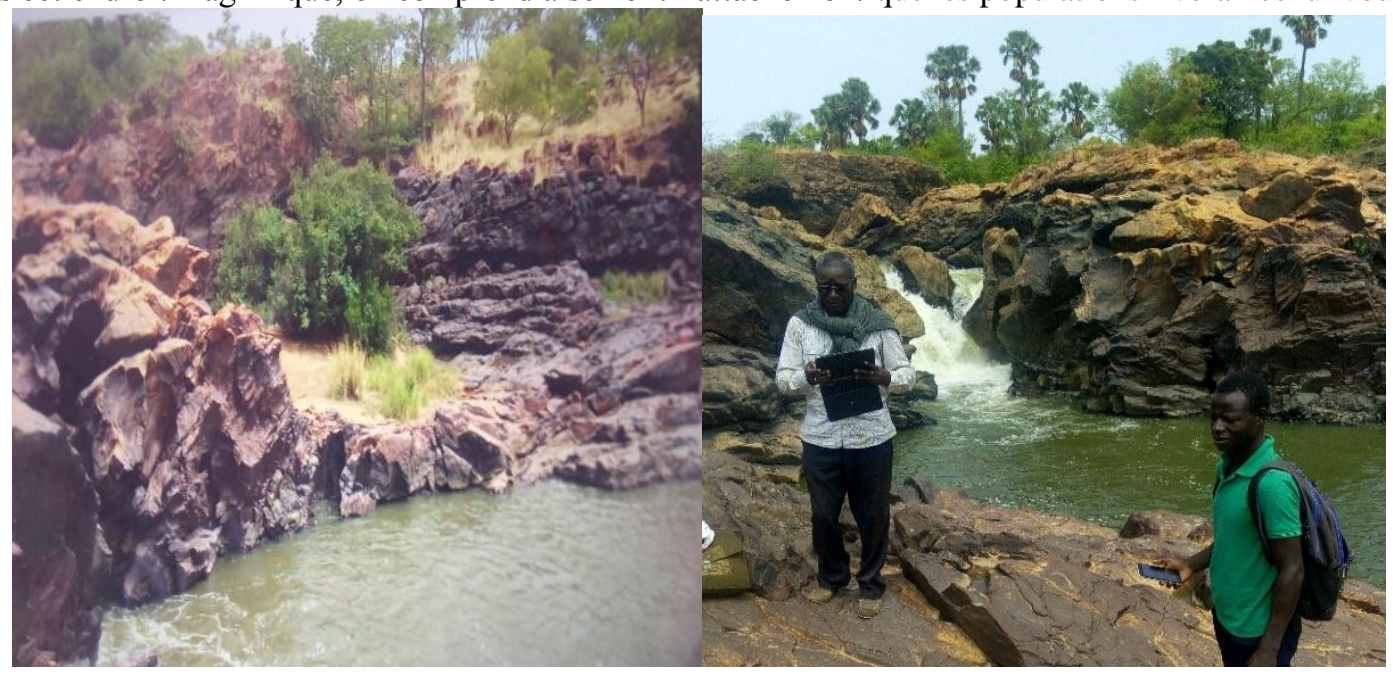

Planche 3 :-Chute de koudou en mai 2018 


\section{Prise de vue : MOUMOUNI, mai 2018}

La planche 3 présente le fétiche de Bonni (Kérou) appelé Kpétara (les pierres tressées). Ce fétiche est érigé au bord du cours d'eau. Kpétara incarne les dieux protecteurs de la rivière Mékrou en raison de sa générosité à l'endroit des populations riveraines. La photo 3 montre un fétiche Kpétara (les pierres tressées) de Bonni (Kérou).

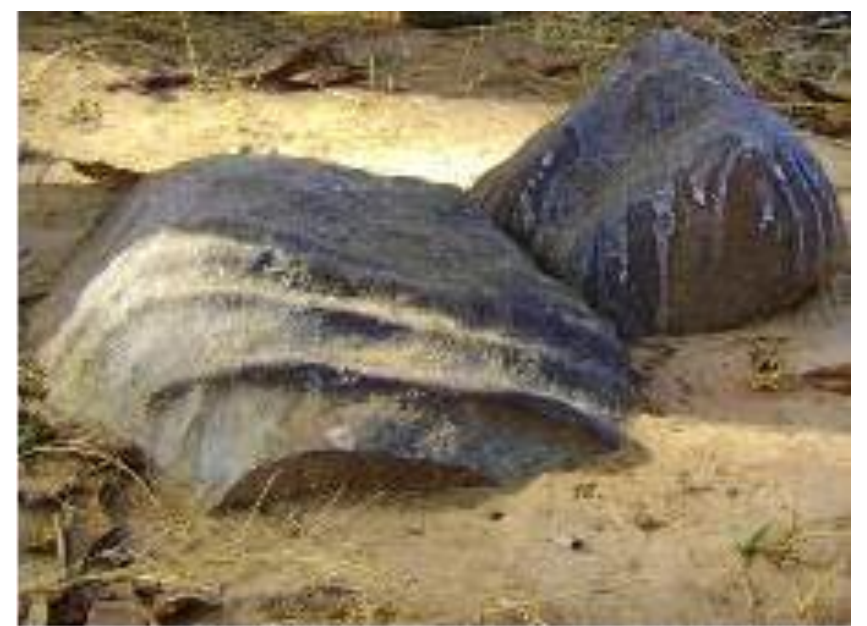

Photo 3 :-Fétiche Kpétara (les pierres tressées) de Bonni (Kérou

\section{Prise De Vue : Moumouni, Mai 2018}

Aussi, les ustensiles de cuisines ne sont-ils pas admis au bord du cours d'eau pour y être lavés. L'accent y est mis sur la cuillère traditionnelle en calebasse qu'utilisent les peulhs pour boire de la bouillie. Par ailleurs, selon les enquêtés, que ce soit en période de hautes eaux ou en période d'étiage, la mékrou ne tolère point la traversée, ou par pirogue ou à pied, d'objets volés.

Autrefois, ces règles endogènes de protection des ressources en eau étaient scrupuleusement respectées et ce, jusqu'à un passé récent, ont laissé entendre les populations. Mais aujourd'hui, les données ont changé. La croissance de la population, l'individualisme, la course effrénée pour le profit, la prolifération des croyances, et la disparition de points, plans et cours d'eau, due aux changements climatiques et aux actions anthropiques, infléchissent la pratique des savoirs endogènes. On assiste à un abandon du patrimoine culturel sacré et au non-respect des interdits.

« La croissance de la population ainsi que la transformation des modes de vie et le développement économique dans les pays en développement en générale...... ont accentué la pression sur les ressources en eau. Les problèmes environnementaux viennent s'ajouter à ces pressions ». (BOKO, 2001) cité par (HEDIBLE, 2007 p 30).

Cependant, on lit aisément, au travers de leurs réflexes comportementaux, que les populations ignorent allègrement tout du dispositif moderne, juridico-institutionnel, mis en place en faveur de la jouissance et de la gestion rationnelle de ces ressources en eau.

\section{Effet de la culture du coton sur la ressource eau}

Dans le bassin, la culture du coton est l'activité agricole qui occupe le plus les différents communautés. Le coton dont le Bénin tire l'essentiel de ses revenus, représente à lui seul près des trois quarts des recettes d'exportation. Or, le cotonnier est la plante la plus parasitée au monde. Sa culture est grande consommatrice de produits chimiques (engrais chimique, pesticides, herbicide) qui hypothèquent la qualité des eaux du bassin. Quant à la quantité de ces ressources, elle est menacée par des techniques culturales qui occasionnent la destruction du couvert végétal et l'abattage incontrôlé des ligneux par les exploitants forestiers auxquels l'Etat délivre des permis ou des agréments. Les gardes forêts seraient complices d'un tel désastre. Les quantités de ces ressources sont également menacées par les changements climatiques.

\section{Discussion:-}

Pour les populations du bassin de la Mékrou, l'eau a une dimension à la fois culturelle et cultuelle. Elle abriterait des divinités. L'eau revêt un caractère sacré et sa protection passe par le recours à ces divinités au travers des 
invocations, de la pratique des rituels, des cultes et de l'instauration des interdits etc. Ces résultats corroborent ceux des travaux de (Djibril DEBOUROU, 2012, p 319) réalisés dans sa thèse de doctorat.

Les règles modernes de gestion des ressources en eau ne sont pas, en l'état actuel des choses, (l'analphabétisme des populations foncièrement ancrées dans leurs réalités socio-culturelles) ne pourront pas assurer leurs réelles protections. Ce résultat confirme les résultats de (GLEauBe, 2012).

Autrefois, les règles endogènes de protection des ressources en eau étaient scrupuleusement respectées et ce, jusqu'à un passé récent, Mais aujourd'hui, les données ont changé. La croissance de la population, l'individualisme, la course effrénée pour le profit, la prolifération des croyances, et la disparition de points, plans et cours d'eau, due aux changements climatiques et aux actions anthropiques, infléchissent la pratique des savoirs endogènes. C'est le constat de BOKO, 2001 cités par (HEDIBLE, 2007 p 30).

\section{Conclusion:-}

En raison de son importance vitale et de son caractère sacré, l'eau a, de tout temps, bénéficié de la protection des populations du bassin de la Mékrou au travers de pratiques relevant de leurs savoirs endogènes. Aujourd'hui, plusieurs facteurs infléchissent l'observance de ces règles traditionnelles. Aussi, lit-on aisément, au travers de leurs réflexes comportementaux, que ces populations ignorent allègrement tout du dispositif moderne, juridicoinstitutionnel, mis en place en faveur de la jouissance et de la gestion rationnelle de ces ressources en eau.

Le manque de sensibilisation des populations du bassin, analphabètes à près de quatre-vingt- dix pour cent, autour des lois votées en faveur des ressources en eau, explique que les règles modernes ne puissent pas leur assurer une véritable protection. Aussi, ces règles modernes devraient être associées aux stratégies endogènes de protection afin de leur garantir une gestion plus rationnelle et plus durable.

\section{References:-}

1. Ali MOUMOUNI BAWA N'GOBI, (2007) : Le droit de l'eau au Bénin. Mémoire de DEA en droit, Université d'Abomey- Calavi, Cotonou, Bénin, $60 \mathrm{p}$.

2. Aymeric PICQUE, (2016) : Evaluation de l'impact du Glyphosate sur la santé humaine. Thèse de doctorat en pharmacie, $66 \mathrm{p}$

3. Bruno HELLENDORFF (2013) : l'eau, les conflits et la coopération : Gestion de l'eau en Afrique de l'Ouest : Risques et opportunités. Groupe de Recherche et d'Information sur la Paix et la sécurité (GRIP), 18 p.

4. Clarisse S. HEDIBLE (2007) : Perception et stratégies d'adaptation des communautés rurale du département de l'atlantique face à la dégradation de la qualité de l'eau de consommation. Thèse de Doctorat Unique, Université d'Abomey-Calavi. Ecole Doctorale Pluridisciplinaire "Espace, Cultures et Développement", 215 p.

5. Djibril DEBOUROU, (2012) : La société baatonnu du Nord-Bénin son passé, son dynamisme, ses conflits et ses innovations. Thèse de Doctorat Unique, Université d'Abomey-Calavi. Ecole Doctorale Pluridisciplinaire "Espace, Cultures et Développement", 704 p.

6. Expédit Wilfried VISSIN, (2007) : Impact de la variabilité climatique et de la dynamique des états de surface sur les écoulements du bassin béninois du fleuve Niger. Thèse de Doctorat Unique, Université d'AbomeyCalavi. Ecole Doctorale Pluridisciplinaire "Espace, Cultures et Développement", 310 p.

7. GLEauBe, (2012) Etude portant état des lieux et gestion de l'information sur les ressources en eau dans le bassin de la Mékrou. Rapport final, 104 p.

8. LIFAD, (2006) : Etude des systèmes de gestion/utilisation de l'eau et définition des actions prioritaires de valorisation locale des ressources eau dans une approche gire au bénin Volume 1 - Etat des lieux de la gestion des ressources en eau du Bénin. 121 p.

9. Madiodio Niasse, Alejandro Iza, Amidou Garane et Olli Varis, (2002) : La gouvernance de l'eau en Afrique de l'ouest : aspects juridiques et institutionnels. Atelier sur la gouvernance de l'eau $247 \mathrm{p}$.

10. Michel BOKO, (2004) : Pollution de l'environnement et santé publique. Manuel de cours, $160 \mathrm{p}$.

11. PNE-Bénin (2016): L'eau au service de la croissance et de la lutte contre la pauvreté dans le bassin transfrontalier de la Mékrou. Etude portant Identification des priorités de développement sur la portion du territoire du Bénin se situant dans le bassin transfrontalier de la Mékrou, $166 \mathrm{p}$.

12. PNE-Bénin, (2015) Analyse de l'utilisation actuelle des ressources en eau et définition de la situation de référence sur la portion du territoire du Bénin se situant dans le bassin de la Mékrou Et les études des plans 
GIRE, des politiques et stratégies de croissance verte et de gestion des situations de sécheresse et d'inondation, des politiques et stratégies énergétiques et de la lutte contre la pauvreté au Bénin $171 \mathrm{p}$

13. PNE Bénin, 2015) «L'eau au service de la croissance et la lutte contre pauvreté dans le bassin de la Mékrou projet d'appui à la restauration des écosystèmes de la tête de bassin de la Mékrou (PAREM), 156 p.

14. Vanja Westerberg, (2017) : L'Initiative de l'économie de la dégradation des terres: Une étude de cas de la commune de Banikoara au Bénin. Etude de cas 36 p. 